\title{
新型含噁唑单元的吡唑酰胺衍生物的合成及其生物活性
}

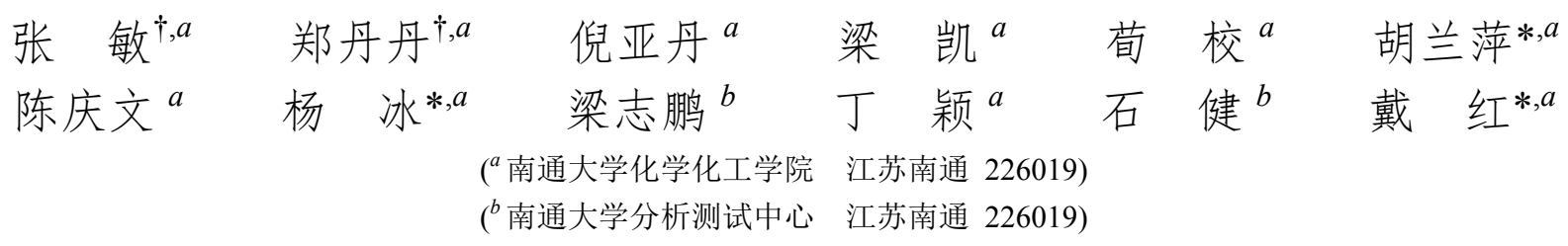

\begin{abstract}
摘要 为了寻找具有良好生物活性的吡唑酰胺化合物, 基于氯虫苯甲酰胺的结构, 在吡唑环的 5-位引入取代腎唑环单 元, 合成出了一系列新型吡唑酰胺, 利用 ${ }^{1} \mathrm{H} N \mathrm{NMR},{ }^{13} \mathrm{C} \mathrm{NMR}$ 和元素分析对其目标产物的结构进行了确证. 生测结果表 明, 所有目标化合物在 $500 \mu \mathrm{g} / \mathrm{mL}$ 时对粘虫均显示出较好的杀虫活性(致死率 $\geqslant 90 \%$ ). 5 个化合物在 $100 \mu \mathrm{g} / \mathrm{mL}$ 时对粘虫 的防治效果可达 $90 \% \sim 100 \%, 3$ 个化合物在 $100 \mu \mathrm{g} / \mathrm{mL}$ 时对苜葆蜴的杀死率为 $90 \% \sim 100 \%$. 另外, 2 个化合物在 500 $\mu \mathrm{g} / \mathrm{mL}$ 时对朱砂叶螨的防效可达 $80 \% \sim 100 \%$.
\end{abstract}

关键词 噁唑; 吡唑酰胺; 合成; 生物活性

\section{Synthesis and Bioactivities of Novel Pyrazole Amides Carrying Oxazole Moiety}

\author{
Zhang, Min ${ }^{\dagger, a} \quad$ Zheng, Dandan ${ }^{\dagger, a} \quad \mathrm{Ni}$, Yadan $^{a} \quad$ Liang, Kai $^{a} \quad$ Xun, Xiao ${ }^{a}$ \\ $\mathrm{Hu}$, Lanping ${ }^{*, a} \quad$ Chen, Qingwen $^{a} \quad$ Yang, Bing $^{*, a} \quad$ Liang, Zhipeng ${ }^{b}$ \\ Ding, Ying ${ }^{a}$ Shi, Jian ${ }^{b}$ Dai, Hong ${ }^{*, a}$ \\ $\left({ }^{a}\right.$ College of Chemistry and Chemical Engineering, Nantong University, Nantong, Jiangshu 226019) \\ ( ${ }^{b}$ Analysis and Testing Center, Nantong University, Nantong, Jiangshu 226019)
}

\begin{abstract}
In order to find new pyrazole amides with wonderful bioactivities, a series of novel pyrazole amide derivatives were synthesized by introducing substituted oxazole ring into the C-5 position of pyrazole skeleton based on the lead chlorantraniliprole. The aimed compounds were structurally characterized through ${ }^{1} \mathrm{H}$ NMR, ${ }^{13} \mathrm{C}$ NMR and elemental analysis. The preliminary bioassay results exhibited that all the title compounds displayed more than $90 \%$ insecticidal activities against Mythimna separata (Walker) at $500 \mu \mathrm{g} / \mathrm{mL}$. At the dosage of $100 \mu \mathrm{g} / \mathrm{mL}$, five compounds possessed $90 \% \sim 100 \%$ insecticidal activities against Mythimna separata (Walker), and three compounds exhibited insecticidal property against Aphis craccivora with $90 \% \sim 100 \%$. Additionally, at the dosage of $500 \mu \mathrm{g} / \mathrm{mL}$, two compounds possessed insecticidal activity against Tetranychus cinnabarinus with $80 \% \sim 100 \%$.
\end{abstract}

Keywords oxazole; pyrazole amide; synthesis; bioactivity

噁唑是含有氮、氧原子的五元芳香性杂环. 含噁唑 环化合物在农药和医药领域具有杀虫 ${ }^{[1]} 、$ 除草 ${ }^{[2]} 、$ 杀菌 ${ }^{[3]}$ 、 抗癌 ${ }^{[4]}$ 等活性. 如 Wang 等 ${ }^{[5]}$ 研制的化合物 $\mathbf{A}$ (图 1)在测 试剂量为 $50 \mathrm{mg} / \mathrm{L}$ 时对粘虫的杀灭活性为 $70 \%$, Shen 课 题组 ${ }^{[6]}$ 研究发现化合物 B(图 1)在测试剂量为 $500 \mu \mathrm{g} / \mathrm{mL}$ 时对粘虫、蚜虫和螨虫均有 $100 \%$ 的杀灭效果; $\mathrm{Fu}$ 等 ${ }^{[7]}$
报道的化合物 $\mathbf{C}$ (图 1)在测试剂量为 $20 \mathrm{mg} / \mathrm{kg}$ 时对玉米 植株鲜重的恢复效果为 $95.45 \%$. 吡唑酰胺作为含氮杂 环体系中的重要一员, 在农业生产与医疗保健方面扮演 着重要的角色, 在杀虫、杀菌、除草及抗癌等领域 $\left.{ }^{[8} 14\right]$ 有着广泛的应用, 如日本三菱化学公司相继研制出的吡 螨胺(Tebufenpyrad)与唑虫酰胺(Tolfenpyrad)(图 1), 它们

\footnotetext{
* Corresponding authors. E-mail: hlp@ntu.edu.cn; yangbing111@ntu.edu.cn; daihong_2015@aliyun.com Received January 20, 2020; revised February 27, 2020; published online March 6, 2020.

Project supported by the National Natural Science Foundation of China (No. 21372135) and the Science and Technology Project Fund of Nantong City (No. MS12019060).

国家自然科学基金(No. 21372135)、南通市科技计划(No. MS12019060)资助项目.

共同第一作者(These authors contributed equally to this work).
} 
常常被用于防治附线螨、小菜蛾等害虫 ${ }^{[15,16]}$. 近年来, 一些科研工作者在此基础上衍生制备出了许多具有新 颖结构的吡唑酰胺衍生物. 如美国杜邦公司成功开发的 氯虫苯甲酰胺(Chlorantraniliprole, 图 1)有杀虫谱广、毒 性低等特点, 对粘虫和小菜蛾等害虫都表现出良好的杀 虫作用 ${ }^{[17]}$; Kang 等 ${ }^{[18]}$ 基于氯虫苯甲酰胺的结构制备出 的化合物 $\mathbf{D}$ (图 1)对小菜蛾呈现优异的杀灭活性, 其 $\mathrm{LC}_{50}$ 值为 $2.04 \mu \mathrm{g} / \mathrm{mL}$; Zhu 等 ${ }^{[19]}$ 报道的化合物 $\mathbf{E}$ (图 1)对 粘虫显示出良好的杀虫效果; Wang 课题组 ${ }^{[20]}$ 研发的化 合物 $\mathbf{F}$ (图 1) 表现出较好的杀虫和杀菌活性, 化合物 $\mathbf{F}$ 在 $200 \mu \mathrm{g} / \mathrm{mL}$ 测试剂量下对粘虫的杀灭活性为 $100 \%$, 在 $50 \mu \mathrm{g} / \mathrm{mL}$ 测试剂量下对苹果轮纹病菌的抑制效果达 $89.7 \%$. 因此, 本课题组基于商品化的杀虫剂氯虫苯甲 酰胺的结构, 将取代噁唑环通过亚甲基桥引入吡唑酰胺 分子结构中, 设计合成了一系列新颖的吡唑酰胺化合物 (图 2), 同时测试了目标产物的杀虫活性, 生物活性测试
结果显示, 部分目标化合物呈现出较好的杀虫作用. 目 标化合物的合成路线见 Scheme 1.

\section{1 结果与讨论}

\section{1 目标化合物的合成}

在目标化合物 11 的制备时，选取取代基为 4-Cl 的 化合物 11d 为例，以中间体 $5 \mathbf{d}$ 和 10 为原料，探索了不 同的溶剂和缚酸剂对化合物 11d 收率的影响(表 1). 在试 验过程中发现, 当选取二氯甲烷作为反应溶剂, 三乙胺 作为缚酸剂, 室温搅拌 $12 \mathrm{~h}$, 反应转化率相对较佳, 化 合物 11d 收率为 $70 \%$, 同时后续处理也较为简便. 因此, 选用该方法顺利合成了其它目标化合物.

\section{2 目标产物的核磁数据剖析}

以化合物 $11 \mathrm{a}$ 为例, 对其 ${ }^{1} \mathrm{H}$ NMR 和 ${ }^{13} \mathrm{C} \mathrm{NMR}$ 数据 进行剖析. 从化合物 11a 的 ${ }^{1} \mathrm{H}$ NMR 数据可发现, 啞唑<smiles>CNC(=O)c1cc(Cl)cc(C)c1NC(=O)c1coc(-c2ccc(Cl)cc2)n1</smiles>

A<smiles>CSc1nn(C)c(C(=O)NCc2ccc(C(C)(C)C)cc2)c1Cl</smiles>

Tebufenpyrad<smiles>O=C(CNC(=O)c1cc(Br)nn1-c1ncccc1Cl)Nc1ccccc1[N+](=O)[O-]</smiles><smiles>Cc1nn(C)c(Oc2ccc(F)cc2)c1/C=N/OCc1ccc(-c2cnco2)cc1</smiles><smiles>CCc1nn(C)c(C(=O)NCc2ccc(Oc3ccc(C)cc3)cc2)c1Cl</smiles><smiles>CC(C)NC(=O)c1ccc(Cl)cc1N(O)C(=O)c1cc(Br)nn1-c1ncccc1Cl</smiles><smiles>Cc1oc(CC2(C)OCCO2)nc1-c1ccc(F)cc1</smiles>

c<smiles>CNC(=O)c1cc(Cl)cc(C)c1NC(=O)c1cc(Br)nn1-c1ncccc1Cl</smiles><smiles>Cc1cc(Cl)cc(C(=O)N(C)C)c1N1CC1c1cc(Br)nn1-c1ncccc1Cl</smiles>

图 1 化合物 $\mathbf{A} \sim \mathbf{F}$ 、吡螨胺、唑虫酰胺和氯虫苯甲酰胺的化学结构

Figure 1 Chemical structures of compounds $\mathbf{A} \sim \mathbf{F}$, tebufenpyrad, tolfenpyrad and chlorantraniliprole<smiles>CNC(=O)c1cc(Cl)cc(I)c1NC(=O)c1cc(Br)nn1-c1ncccc1Cl</smiles>

Chlorantraniliprole

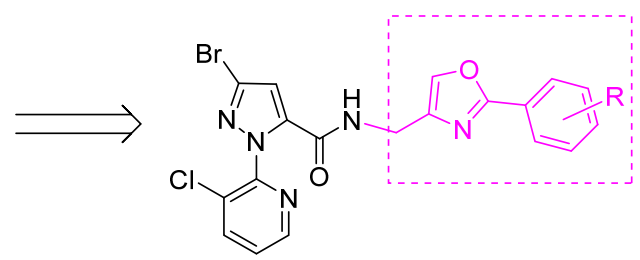

11

图 2 目标化合物 $\mathbf{1 1}$ 的分子设计

Figure 2 Design strategy of the title compounds 11 
<smiles>[R][R]1ccc(C(=O)Cl)cc1</smiles><smiles></smiles><smiles>CCCCC(=O)c1cc(Br)nn1-c1ncc[13c]c1Cl</smiles>

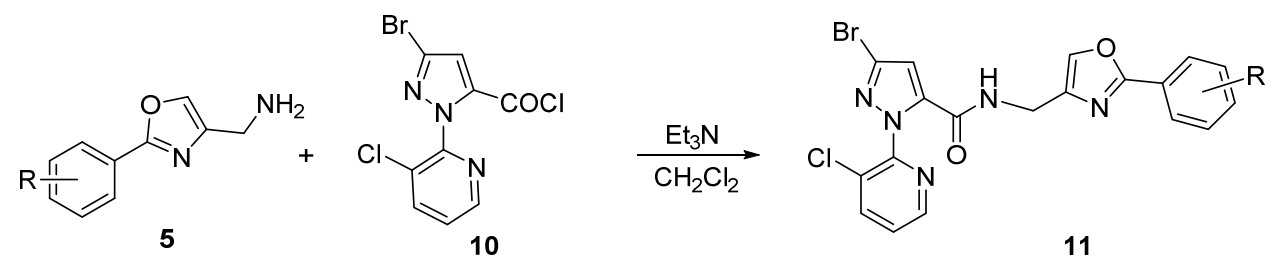

11a: $R=3-F, 11 b: R=2-C l, 11 c: R=3-C l, 11 d: R=4-C l, 11 e: R=3-B r, 11 f: R=4-B r ; 11 g: R=2-C F_{3}, 11 h: R=4-C F_{3}, 11 i: R=4-C N, 11 j: R^{1}=$ $4-\mathrm{CH}_{3}, 11 \mathrm{k}: \mathrm{R}=3-\mathrm{CH}_{3}-4-\mathrm{NO}_{2}, 11 \mathrm{I}: \mathrm{R}=3-\mathrm{NO}_{2}-4-\mathrm{CH}_{3}, 11 \mathrm{~m}: \mathrm{R}=2-\mathrm{F}-6-\mathrm{Cl}, 11 \mathrm{n}: \mathrm{R}=2,4-\mathrm{Cl}_{2} ; 11 \mathrm{o}: \mathrm{R}=3,5-\mathrm{Cl}_{2} ; 11 \mathrm{p}: \mathrm{R}=2,6-\mathrm{F}_{2}$

图式 1 目标化合物 11 的合成路线

Scheme 1 Synthetic route of the target compounds 11

表 1 反应条件对化合物 11d 收率的影响 Table 1 Effects of reaction conditions on the synthesis of compound 11d

\begin{tabular}{|c|c|c|c|c|}
\hline Entry & Base & Solvent & Reaction condition & Yield/\% \\
\hline 1 & Pyridine & $\mathrm{CH}_{3} \mathrm{COCH}_{3}$ & r.t. for $12 \mathrm{~h}$ & 20 \\
\hline 2 & Pyridine & $\mathrm{CH}_{2} \mathrm{Cl}_{2}$ & r.t. for $12 \mathrm{~h}$ & 61 \\
\hline 3 & Pyridine & THF & r.t. for $12 \mathrm{~h}$ & 33 \\
\hline 4 & Pyridine & $\mathrm{CH}_{3} \mathrm{CN}$ & r.t. for $12 \mathrm{~h}$ & 52 \\
\hline 5 & Pyridine & $\mathrm{CH}_{2} \mathrm{Cl}_{2}$ & Reflux for $12 \mathrm{~h}$ & 56 \\
\hline 6 & Pyridine & $\mathrm{CH}_{3} \mathrm{CN}$ & Reflux for $12 \mathrm{~h}$ & 45 \\
\hline 7 & $\mathrm{Et}_{3} \mathrm{~N}$ & $\mathrm{CH}_{2} \mathrm{Cl}_{2}$ & r.t. for $12 \mathrm{~h}$ & 70 \\
\hline 8 & $\mathrm{Et}_{3} \mathrm{~N}$ & THF & r.t. for $12 \mathrm{~h}$ & 43 \\
\hline 9 & $\mathrm{Et}_{3} \mathrm{~N}$ & $\mathrm{CH}_{3} \mathrm{CN}$ & r.t. for $12 \mathrm{~h}$ & 59 \\
\hline 10 & $\mathrm{Et}_{3} \mathrm{~N}$ & $\mathrm{CH}_{2} \mathrm{Cl}_{2}$ & Reflux for $12 \mathrm{~h}$ & 63 \\
\hline 11 & $\mathrm{Et}_{3} \mathrm{~N}$ & $\mathrm{CH}_{3} \mathrm{CN}$ & Reflux for $12 \mathrm{~h}$ & 50 \\
\hline 12 & $\mathrm{~K}_{2} \mathrm{CO}_{3}$ & $\mathrm{CH}_{2} \mathrm{Cl}_{2}$ & r.t. for $12 \mathrm{~h}$ & 12 \\
\hline 13 & $\mathrm{KOH}$ & $\mathrm{CH}_{2} \mathrm{Cl}_{2}$ & r.t. for $12 \mathrm{~h}$ & 0 \\
\hline
\end{tabular}

环上的氢在 $\delta 7.62$ 以单峰形式出现, 3-F 取代苯环上两个 氢在 $\delta 7.80$ 和 7.70 分别以双重峰形式出现, 3-F 取代苯 环上其它两个氢在 $\delta 7.48 \sim 7.44$ 和 $7.21 \sim 7.16$ 分别以多 重峰形式出现, $\mathrm{CONH}$ 上氢的化学位移在 $\delta 7.05$, 取代吡 唑环上 4-位氢在 $\delta 6.81$ 以单峰形式出现, 与 $\mathrm{NH}$ 相连的 $\mathrm{CH}_{2}$ 两个氢在 $\delta 4.45$ 以双重峰形式出现; 从化合物 $11 \mathrm{a}$ 的 ${ }^{13} \mathrm{C}$ NMR 数据数据可知, 酰胺键上羰基碳原子的 $\delta$ 值

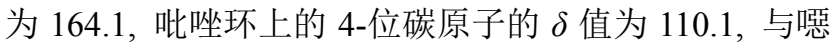
唑环相连的亚甲基碳原子的 $\delta$ 值为 35.3.

\section{3 生物活性}

采用浸叶法 ${ }^{[21]}$ 测试了化合物 $11 \mathrm{a} \sim 11 \mathrm{p}$ 对粘虫 (Mythimna separata) 的杀虫活性，同时利用喷雾法 ${ }^{[22]}$ 测 试了化合物 11a 11p 对朱砂叶螨(Tetranychus cinnabarrinus) 与苜宿蚜 (Aphis craccivora) 的杀虫活性, 其相关数 据如表 2 所示. 
表 2 目标化合物 $11 \mathrm{a} \sim 11 \mathrm{p}$ 的杀虫活性(死亡率/\%) ${ }^{a}$

Table 2 Insecticidal activities (mortality/\%) of title compounds 11a $\sim 11 p$

\begin{tabular}{|c|c|c|c|c|c|c|c|c|}
\hline \multirow{2}{*}{ Compd. } & \multicolumn{3}{|c|}{ Mythimna separata } & \multicolumn{3}{|c|}{ Aphis craccivora } & \multicolumn{2}{|c|}{ Tetranychus cinnabarinus } \\
\hline & $500 \mu \mathrm{g} / \mathrm{mL}$ & $100 \mu \mathrm{g} / \mathrm{mL}$ & $20 \mu \mathrm{g} / \mathrm{mL}$ & $500 \mu \mathrm{g} / \mathrm{mL}$ & $100 \mu \mathrm{g} / \mathrm{mL}$ & $20 \mu \mathrm{g} / \mathrm{mL}$ & $500 \mu \mathrm{g} / \mathrm{mL}$ & $100 \mu \mathrm{g} / \mathrm{mL}$ \\
\hline $11 \mathrm{a}$ & 100 & 0 & - & 0 & - & - & 0 & - \\
\hline $11 \mathrm{~b}$ & 100 & 0 & - & 100 & 90 & 30 & 0 & - \\
\hline $11 \mathrm{c}$ & 100 & 0 & - & 0 & - & - & 0 & - \\
\hline 11d & 100 & 100 & 30 & 0 & - & - & 0 & - \\
\hline $11 \mathrm{e}$ & 90 & 0 & - & 0 & - & - & 0 & - \\
\hline 11f & 100 & 100 & 40 & 0 & - & - & 0 & - \\
\hline $11 \mathrm{~g}$ & 100 & 0 & - & 0 & - & - & 0 & - \\
\hline $11 \mathrm{~h}$ & 100 & 100 & 50 & 100 & 100 & 30 & 100 & 0 \\
\hline $11 \mathrm{i}$ & 100 & 0 & - & 0 & - & - & 0 & - \\
\hline $11 \mathrm{j}$ & 100 & 0 & - & 0 & - & - & 0 & - \\
\hline $11 k$ & 100 & 100 & 40 & 100 & 100 & 20 & 80 & 0 \\
\hline 111 & 100 & 0 & - & 0 & - & - & 0 & - \\
\hline $11 \mathrm{~m}$ & 100 & 0 & - & 0 & - & - & 0 & - \\
\hline $11 n$ & 100 & 0 & - & 0 & - & - & 0 & - \\
\hline 110 & 100 & 90 & 0 & 0 & - & - & 0 & - \\
\hline $11 p$ & 100 & 0 & - & 0 & - & - & 0 & - \\
\hline Tolfenpyrad & 100 & 50 & 40 & - & - & - & - & - \\
\hline Imidacloprid & - & - & - & 100 & 100 & 100 & - & - \\
\hline Fenpyroximate & - & - & - & - & - & - & 100 & 100 \\
\hline
\end{tabular}

化合物 11a 11p 在 $500 \mu \mathrm{g} / \mathrm{mL}$ 时对粘虫都显示出 优异的杀虫作用，化合物 $11 \mathrm{a} \sim 11 \mathrm{p}$ 对粘虫的杀死率为 $90 \% \sim 100 \%$ ，与对照药唑虫酰胺的杀虫效果相接近. 部 分化合物在 $100 \mu \mathrm{g} / \mathrm{mL}$ 时对粘虫的杀死率均在 $90 \%$ 以 上，通过构效关系分析不难发现，当在苯环上引入氯、 溴原子或三氟甲基基团时, 其对位取代物对粘虫的杀虫 活性要优于邻位或间位的杀虫效果，如取代基 $\mathrm{R}$ 为 4- $\mathrm{Cl}$ 的化合物 11d 对粘虫的杀死率为 $100 \%$, 明显高于取代 基 $\mathrm{R}$ 为 $2-\mathrm{Cl}$ 的化合物 $11 \mathrm{~b}$ 和 3- $\mathrm{Cl}$ 的化合物 $11 \mathrm{c}$ 的药效, 当苯环对位含有吸电子基团如氯、澳原子、三氟甲基或 硝基基团时，化合物 11d、11f、11h 和 $11 \mathrm{k}$ 对粘虫的杀 虫活性比苯环对位含有给电子基团甲基的化合物 $11 \mathrm{j}$ 和 111 要好. 在浓度降至 $20 \mu \mathrm{g} / \mathrm{mL}$ 时, 化合物 11d、11f、 $11 \mathrm{~h}$ 和 $11 \mathrm{k}$ 对粘虫仍有 $30 \% \sim 50 \%$ 的杀虫活性，接近于 对照药剂唑虫酰胺的杀虫效果. 同时, 化合物 $11 \mathrm{~b} 、 11 \mathrm{~h}$ 和 $11 \mathrm{k}$ 在 500 和 $100 \mu \mathrm{g} / \mathrm{mL}$ 浓度下对苜葆蚜的杀死率均 超过 $90 \%$, 与对照药剂吡虫啉的杀虫活性相当, 当浓度 降为 $20 \mu \mathrm{g} / \mathrm{mL}$ 时, 化合物 $11 \mathrm{~b} 、 11 \mathrm{~h}$ 和 $11 \mathrm{k}$ 对苜宿蚜还 显示出 $20 \% \sim 30 \%$ 的杀虫作用, 明显优于其它目标化合 物. 此外, 化合物 $11 \mathrm{~h}$ 和 $11 \mathrm{k}$ 在 $500 \mu \mathrm{g} / \mathrm{mL}$ 时对朱砂叶 螨具有 $100 \%$ 和 $80 \%$ 的杀虫效果. 从构效关系分析也能 看出, 目标化合物 $11 \mathrm{~h}\left(\mathrm{R}=4-\mathrm{CF}_{3}\right)$ 和 $11 \mathrm{k}\left(\mathrm{R}=3-\mathrm{CH}_{3}-4-\right.$ $\mathrm{NO}_{2}$ ) 有广谱的杀虫活性, 对所测定的害虫均显示出较 好的杀虫效果, 可作为杀虫先导做进一步的结构优化与 杀虫活性研究.

\section{2 结论}

本研究合成出了 16 个含噁唑单元的新型吡唑酰胺 类化合物. 试验结果表明, 所有化合物在浓度为 500 $\mu \mathrm{g} / \mathrm{mL}$ 时对粘虫的杀死率在 $90 \% \sim 100 \%$, 在 $100 \mu \mathrm{g} / \mathrm{mL}$ 浓度下化合物 11d、11f、11h、11k 和 $11 \mathrm{o}$ 对粘虫的杀虫 活性大于 $90 \%$; 化合物 $11 \mathrm{~b} 、 \mathbf{1 1 h}$ 和 $11 \mathrm{k}$ 在 $100 \mu \mathrm{g} / \mathrm{mL}$ 时对苜宿蚜具有 $90 \%$ 以上的杀虫效果, 在 $20 \mu \mathrm{g} / \mathrm{mL}$ 时 活性不明显; 化合物 $11 \mathrm{~h}$ 和 $11 \mathrm{k}$ 在 $500 \mu \mathrm{g} / \mathrm{mL}$ 时朱砂叶 螨的杀虫活性分别为 $100 \%$ 和 $80 \%$. 本文为今后继续从 事吡唑酰胺类杀虫剂的创制提供了重要的试验数据.

\section{3 实验部分}

\section{1 仪器与试剂}

${ }^{1} \mathrm{H}$ NMR 和 ${ }^{13} \mathrm{C}$ NMR 的数据经 BRUKER $400 \mathrm{MHz}$ 核磁共振仪(TMS 为内标) 测定; 熔点由 X-4 显微熔点测 定仪(温度计未经校正)测试; 元素分析利用 YanacoCHN CORDER MT-3 自动元素分析仪测定. 所用试剂都 是分析纯.

\section{2 化合物 1 2 和 6 10 的合成}

化合物 1 2 的合成参照文献[23], 化合物 6 10 的 合成参照文献[24]和[25].

\section{3 化合物 3 的合成}

将中间体 2 (0.05 mol)及 1,3 -二氯丙酮 $(0.09 \mathrm{~mol})$ 加 
入一反应瓶中, 加完后, 升温至 $130{ }^{\circ} \mathrm{C}$, 反应 $6 \sim 9 \mathrm{~h}$. 反应完成后, 将反应液冷却至室温, 向其加入 $100 \mathrm{~mL}$ 水, 用氯仿萃取数次, 有机层经干燥、过滤、浓缩得到 中间体 3, 直接用于下一步的反应.

为验证化合物 3 的结构, 以取代基 $\mathrm{R}$ 为 $2,4-\mathrm{Cl}_{2}$ 的化 合物作为代表, 通过柱层析分离 $[V$ (乙酸乙酯) : $V$ (石油 醚 $)=1 ： 6$ 得白色固体, 产率 $65 \%$. m.p. $61 \sim 63{ }^{\circ} \mathrm{C} ;{ }^{1} \mathrm{H}$ NMR $\left(\mathrm{CDCl}_{3}, 400 \mathrm{MHz}\right) \delta: 7.94(\mathrm{~d}, J=8.80 \mathrm{~Hz}, 1 \mathrm{H}), 7.78$ (s, 1H), $7.51(\mathrm{~d}, J=2.02 \mathrm{~Hz}, 1 \mathrm{H}), 7.35 \sim 7.32(\mathrm{~m}, 1 \mathrm{H})$, 4.59 (s, $2 \mathrm{H}) ;{ }^{13} \mathrm{C} \mathrm{NMR}\left(\mathrm{CDCl}_{3}, 101 \mathrm{MHz}\right) \delta: 159.4,139.0$, 136.9, 136.8, 133.4, 131.9, 131.0, 127.3, 124.6, 36.9. Anal. calcd for $\mathrm{C}_{10} \mathrm{H}_{6} \mathrm{Cl}_{3} \mathrm{NO}$ : C 45.75, H 2.30, N 5.34; found $\mathrm{C}$ 45.59, H 2.43, N 5.49.

\section{4 化合物 4 的合成}

将中间体 $3(20 \mathrm{mmol}) 、$ 邻苯二甲酰亚胺钾盐 $(36$ $\mathrm{mmol}$ )及 $60 \mathrm{~mL} \mathrm{~N}, \mathrm{~N}$-二甲基甲酰胺(DMF)加入反应瓶中, 加完后室温反应 12 18 h. 反应结束后, 将反应液倒入 盛有 $150 \mathrm{~mL}$ 水的烧杯中, 静置析出固体, 经抽滤、烘干 得中间体 4 , 不经纯化直接用于后面的反应.

\section{5 化合物 5 的合成}

将中间体 $4(10 \mathrm{mmol}) 、 80 \%$ 水合肼 $(30 \mathrm{mmol})$ 及 30 $\mathrm{mL}$ 无水乙醇加入反应瓶中, 加完后, 加热回流反应 6 $8 \mathrm{~h}$, 薄层色谱(TLC)跟踪反应的进度. 反应完成后, 将 反应液冷却至室温, 过滤除去不溶物, 母液旋蒸除去溶 剂得到中间体 $\mathbf{5}$, 不经提纯化直接投入下一步反应.

\subsection{3 -溴-1-(3-氯吡啶-2-基)- $N$-[(2-取代苯基噁唑-4-基)} 甲基]-1H-吡唑-5-甲酰胺(11a 11p)的合成

将中间体 $5(2 \mathrm{mmol})$ 、三乙胺 $(4 \mathrm{mmol})$ 及 $20 \mathrm{~mL}$ 二 氯甲烷加入反应瓶中, 冰浴搅拌下向其滴加 $4 \mathrm{mmol}$ 中 间体 10 的二氯甲烷 $(3 \mathrm{~mL})$ 溶液. 滴加完毕后室温反应 10 18 h. 反应结束后过滤, 母液旋蒸除去溶剂, 得到 的粗品经柱层析分离 $[V$ (乙酸乙酯) $: V$ (石油梄 $)=1: 6]$ 得到目标产物 $11 \mathrm{a} \sim 11 \mathrm{p}$.

3 -溴-1-(3-氯吡啶-2-基)- $N$ - $\{[2$-(3-氟苯基)噁唑-4-基] 甲基\}-1 $H$-吡唑-5-甲酰胺(11a): 白色固体, 产率 63\%. m.p. $183 \sim 185{ }^{\circ} \mathrm{C} ;{ }^{1} \mathrm{H}$ NMR $\left(\mathrm{CDCl}_{3}, 400 \mathrm{MHz}\right) \delta: 8.47$ (d, $J=3.62 \mathrm{~Hz}, 1 \mathrm{H}), 7.90 \sim 7.88(\mathrm{~m}, 1 \mathrm{H}), 7.80$ (d, $J=8.02$ $\mathrm{Hz}, 1 \mathrm{H}), 7.70(\mathrm{~d}, J=9.22 \mathrm{~Hz}, 1 \mathrm{H}), 7.62(\mathrm{~s}, 1 \mathrm{H}), 7.48 \sim$ $7.44(\mathrm{~m}, 1 \mathrm{H}), 7.41 \sim 7.38(\mathrm{~m}, 1 \mathrm{H}), 7.21 \sim 7.16(\mathrm{~m}, 1 \mathrm{H})$, 7.05 (s, 1H), $6.81(\mathrm{~s}, 1 \mathrm{H}), 4.45$ (d, $J=5.22 \mathrm{~Hz}, 2 \mathrm{H}) ;{ }^{13} \mathrm{C}$ NMR $\left(\mathrm{CDCl}_{3}, 101 \mathrm{MHz}\right) \delta: 164.1,161.7,161.0,157.5$, $148.9,146.8,139.2,137.7,136.1,130.7,129.2,128.8$, $128.0,125.8,122.2,117.8,113.4,110.1,35.3$. Anal. calcd for $\mathrm{C}_{19} \mathrm{H}_{12} \mathrm{BrClFN}_{5} \mathrm{O}_{2}$ : C 47.87, $\mathrm{H}$ 2.54, N 14.69; found $\mathrm{C}$ 47.71, H 2.39, N 14.82 .

3 -溴-1-(3-氯吡啶-2-基)- $N$-\{[2-(2-氯苯基)噁唑-4-基] 甲基\}-1H-吡唑-5-甲酰胺(11b)：黄色固体，产率 $62 \%$. m.p. $121 \sim 123{ }^{\circ} \mathrm{C} ;{ }^{1} \mathrm{H}$ NMR $\left(\mathrm{CDCl}_{3}, 400 \mathrm{MHz}\right) \delta: 8.48 \sim$ $8.46(\mathrm{~m}, 1 \mathrm{H}), 8.00 \sim 7.96(\mathrm{~m}, 1 \mathrm{H}), 7.89 \sim 7.87(\mathrm{~m}, 1 \mathrm{H})$, $7.65(\mathrm{~s}, 1 \mathrm{H}), 7.49 \sim 7.19(\mathrm{~m}, 5 \mathrm{H}), 6.80(\mathrm{~s}, 1 \mathrm{H}), 4.46(\mathrm{~d}, J=$ $5.62 \mathrm{~Hz}, 2 \mathrm{H}) ;{ }^{13} \mathrm{C}$ NMR $\left(\mathrm{CDCl}_{3}, 101 \mathrm{MHz}\right) \delta: 161.2$, $158.7,158.4,157.5,148.9,146.8,139.2,137.6,132.5$, $129.5,129.2,127.9,125.8,124.5,117.0,115.3,110.1$, 35.3. Anal. calcd for $\mathrm{C}_{19} \mathrm{H}_{12} \mathrm{BrCl}_{2} \mathrm{~N}_{5} \mathrm{O}_{2}: \mathrm{C} 46.28, \mathrm{H} 2.45, \mathrm{~N}$ 14.20; found C 46.40, H 2.30, N 14.09 .

3-溴-1-(3-氯吡啶-2-基)- $N$-\{[2-(3-氯苯基)噁唑-4-基 $]$ 甲基\}-1H-吡唑-5-甲酰胺(11c)：白色固体，产率 65\%. m.p. $177 \sim 179{ }^{\circ} \mathrm{C} ;{ }^{1} \mathrm{H}$ NMR $\left(\mathrm{CDCl}_{3}, 400 \mathrm{MHz}\right) \delta: 8.48 \sim$ $8.46(\mathrm{~m}, 1 \mathrm{H}), 8.02(\mathrm{~s}, 1 \mathrm{H}), 7.93 \sim 7.88(\mathrm{~m}, 2 \mathrm{H}), 7.63(\mathrm{~s}$, $1 \mathrm{H}), 7.48 \sim 7.39(\mathrm{~m}, 3 \mathrm{H}), 6.94(\mathrm{~s}, 1 \mathrm{H}), 6.83(\mathrm{~s}, 1 \mathrm{H}), 4.46$ (d, $J=5.20 \mathrm{~Hz}, 2 \mathrm{H}) ;{ }^{13} \mathrm{C}$ NMR $\left(\mathrm{CDCl}_{3}, 101 \mathrm{MHz}\right) \delta$ : $160.9,157.4,148.9,146.8,139.2,137.5,136.1,135.1$, $131.0,130.3,129.2,128.2,128.0,126.6,125.8,124.6$, 110.2, 35.2. Anal. calcd for $\mathrm{C}_{19} \mathrm{H}_{12} \mathrm{BrCl}_{2} \mathrm{~N}_{5} \mathrm{O}_{2}$ : C $46.28, \mathrm{H}$ 2.45, N 14.20; found C 46.12, H 2.59, N 14.31.

3 -澳-1-(3-氯吡啶-2-基)- $N$ - $\{$ [2-(4-氯苯基)噁唑-4-基 $]$ 甲基\}-1H-吡唑-5-甲酰胺(11d)：白色固体，产率 70\%. m.p. $169 \sim 171{ }^{\circ} \mathrm{C} ;{ }^{1} \mathrm{H}$ NMR $\left(\mathrm{CDCl}_{3}, 400 \mathrm{MHz}\right) \delta: 8.46$ $(\mathrm{d}, J=3.62 \mathrm{~Hz}, 1 \mathrm{H}), 7.95(\mathrm{~d}, J=8.81 \mathrm{~Hz}, 2 \mathrm{H}), 7.90 \sim 7.87$ $(\mathrm{m}, 1 \mathrm{H}), 7.61(\mathrm{~s}, 1 \mathrm{H}), 7.46(\mathrm{~d}, J=8.42 \mathrm{~Hz}, 2 \mathrm{H}), 7.41 \sim$ $7.38(\mathrm{~m}, 1 \mathrm{H}), 7.06(\mathrm{~s}, 1 \mathrm{H}), 6.82(\mathrm{~s}, 1 \mathrm{H}), 4.45$ (d, $J=5.62$ $\mathrm{Hz}, 2 \mathrm{H}) ;{ }^{13} \mathrm{C} \mathrm{NMR}\left(\mathrm{CDCl}_{3}, 101 \mathrm{MHz}\right) \delta: 161.3,157.4$, $148.9,146.8,139.2,139.1,137.5,137.2,135.9,129.3$, $129.2,128.0,127.8,125.8,125.2,110.1,35.2$. Anal. calcd for $\mathrm{C}_{19} \mathrm{H}_{12} \mathrm{BrCl}_{2} \mathrm{~N}_{5} \mathrm{O}_{2}$ : C 46.28, $\mathrm{H} 2.45, \mathrm{~N} 14.20$; found $\mathrm{C}$ $46.43, \mathrm{H} 2.38, \mathrm{~N} 14.36$.

3-澳-1-(3-氯吡啶-2-基)- $N$ - $\{$ [2-(3-溴苯基)噁唑-4-基 $]$ 甲基\}-1H-吡唑-5-甲酰胺(11e): 黄色固体，产率 64\%. m.p. $166 \sim 168{ }^{\circ} \mathrm{C} ;{ }^{1} \mathrm{H}$ NMR $\left(\mathrm{CDCl}_{3}, 400 \mathrm{MHz}\right) \delta: 8.47 \sim$ $8.45(\mathrm{~m}, 1 \mathrm{H}), 8.16(\mathrm{~s}, 1 \mathrm{H}), 7.94 \sim 7.88(\mathrm{~m}, 2 \mathrm{H}), 7.62 \sim$ $7.59(\mathrm{~m}, 2 \mathrm{H}), 7.41 \sim 7.35(\mathrm{~m}, 2 \mathrm{H}), 7.05(\mathrm{~s}, 1 \mathrm{H}), 6.81(\mathrm{~s}$, $1 \mathrm{H}), 4.44(\mathrm{~d}, J=5.21 \mathrm{~Hz}, 2 \mathrm{H}) ;{ }^{13} \mathrm{C} \mathrm{NMR}\left(\mathrm{CDCl}_{3}, 101\right.$ MHz) $\delta: 160.5,157.5,148.9,146.8,139.3,139.1,137.6$, $136.2,133.9,130.5,129.4,128.5,128.0,125.9,125.0$, 123.0, 110.2, 35.2. Anal. calcd for $\mathrm{C}_{19} \mathrm{H}_{12} \mathrm{Br}_{2} \mathrm{ClN}_{5} \mathrm{O}_{2}$ : C 42.45, H 2.25, N 13.03; found C 42.59, H 2.32, N 13.16.

3 -澳-1-(3-氯吡啶-2-基)- $N$-\{[2-(4-溴苯基)噁唑-4-基 $]$ 
甲基\}-1H-吡坐-5-甲酰胺(11f)：白色固体，产率 73\%. m.p. $195 \sim 197{ }^{\circ} \mathrm{C} ;{ }^{1} \mathrm{H}$ NMR $\left(\mathrm{CDCl}_{3}, 400 \mathrm{MHz}\right) \delta: 8.47 \sim$ $8.45(\mathrm{~m}, 1 \mathrm{H}), 7.89 \sim 7.87(\mathrm{~m}, 3 \mathrm{H}), 7.63 \sim 7.61(\mathrm{~m}, 3 \mathrm{H})$, $7.41 \sim 7.39(\mathrm{~m}, 1 \mathrm{H}), 7.02(\mathrm{~s}, 1 \mathrm{H}), 6.82(\mathrm{~s}, 1 \mathrm{H}), 4.45(\mathrm{~d}, J=$ $5.22 \mathrm{~Hz}, 2 \mathrm{H}) ;{ }^{13} \mathrm{C}$ NMR $\left(\mathrm{CDCl}_{3}, 101 \mathrm{MHz}\right) \delta: 161.3$, $157.4,148.9,146.8,139.2,139.0,137.5,135.9,132.2$, 129.2, 128.0, 127.9, 125.8, 125.6, 125.5, 110.1, 35.3. Anal. calcd for $\mathrm{C}_{19} \mathrm{H}_{12} \mathrm{Br}_{2} \mathrm{ClN}_{5} \mathrm{O}_{2}$ : C 42.45, H 2.25, N 13.03; found $\mathrm{C} 42.28, \mathrm{H} 2.12, \mathrm{~N} 12.88$.

3 -溴-1-(3-氯吡啶-2-基)- $N$-\{ [2-(2-三氟甲基苯基)噁 唑-4-基]甲基\}-1 $H$-吡唑-5-甲酰胺(11g): 黄色固体, 产率 $60 \%$. m.p. $133 \sim 135{ }^{\circ} \mathrm{C} ;{ }^{1} \mathrm{H}$ NMR $\left(\mathrm{CDCl}_{3}, 400 \mathrm{MHz}\right) \delta$ : $8.47 \sim 8.45(\mathrm{~m}, 1 \mathrm{H}), 7.95(\mathrm{~d}, J=7.61 \mathrm{~Hz}, 1 \mathrm{H}), 7.90 \sim 7.87$ (m, 1H), $7.83(\mathrm{~d}, J=7.62 \mathrm{~Hz}, 1 \mathrm{H}), 7.70(\mathrm{~s}, 1 \mathrm{H}), 7.68 \sim$ $7.60(\mathrm{~m}, 2 \mathrm{H}), 7.41 \sim 7.37(\mathrm{~m}, 1 \mathrm{H}), 7.17(\mathrm{~s}, 1 \mathrm{H}), 6.79(\mathrm{~s}$, $1 \mathrm{H}), 4.48(\mathrm{~d}, J=5.62 \mathrm{~Hz}, 2 \mathrm{H}) ;{ }^{13} \mathrm{C}$ NMR $\left(\mathrm{CDCl}_{3}, 101\right.$ MHz) $\delta$ : 160.1, 157.5, 148.9, 146.8, 139. 2, 137.6, 137.0, $132.0,131.5,130.7,129.2,128.7,128.0,127.0,125.8$, 125.6, 124.7, 122.0, 110.1, 35.2. Anal. calcd for $\mathrm{C}_{20} \mathrm{H}_{12} \mathrm{Br}-$ $\mathrm{ClF}_{3} \mathrm{~N}_{5} \mathrm{O}_{2}$ : C 45.61, H 2.30, N 13.30; found $\mathrm{C} 45.50, \mathrm{H}$ 2.43, N 13.46 .

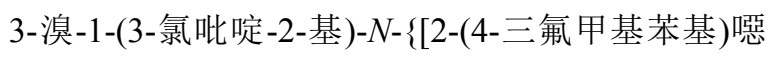
唑-4-基]甲基\}-1 $H$-吡唑-5-甲酰胺(11h): 白色固体, 产率 $65 \%$. m.p. $170 \sim 172{ }^{\circ} \mathrm{C} ;{ }^{1} \mathrm{H}$ NMR $\left(\mathrm{CDCl}_{3}, 400 \mathrm{MHz}\right) \delta$ : $8.48 \sim 8.46(\mathrm{~m}, 1 \mathrm{H}), 8.16(\mathrm{~d}, J=8.02 \mathrm{~Hz}, 2 \mathrm{H}), 7.90 \sim 7.88$ (m, 1H), $7.75(\mathrm{~d}, J=8.41 \mathrm{~Hz}, 2 \mathrm{H}), 7.68(\mathrm{~s}, 1 \mathrm{H}), 7.42 \sim$ 7.39 (m, 1H), 6.97 (s, 1H), 6.84 (s, 1H), 4.49 (d, $J=5.61$ $\mathrm{Hz}, 2 \mathrm{H}) ;{ }^{13} \mathrm{C} \mathrm{NMR}\left(\mathrm{CDCl}_{3}, 101 \mathrm{MHz}\right) \delta: 160.8,157.5$, $148.9,146.8,139.2,139.0,137.7,136.5,129.7,129.2$, 128.0, 126.8, 126.0, 125.8, 110.1, 35.2. Anal. calcd for $\mathrm{C}_{20} \mathrm{H}_{12} \mathrm{BrClF}_{3} \mathrm{~N}_{5} \mathrm{O}_{2}$ : C 45.61, H 2.30, N 13.30; found $\mathrm{C}$ 45.78, H 2.39, N 13.15 .

3-澳-1-(3-氯吡啶-2-基)- $N$ - $\{$ [2-(4-氰基苯基)噁唑-4基]甲基\}- $1 H$-吡唑-5-甲酰胺(11i): 黄色固体, 产率 68\%. m.p. $176 \sim 178{ }^{\circ} \mathrm{C} ;{ }^{1} \mathrm{H}$ NMR $\left(\mathrm{CDCl}_{3}, 400 \mathrm{MHz}\right) \delta: 8.47 \sim$ $8.45(\mathrm{~m}, 1 \mathrm{H}), 8.11(\mathrm{~d}, J=8.41 \mathrm{~Hz}, 2 \mathrm{H}), 7.91 \sim 7.88(\mathrm{~m}$, 1H), $7.77(\mathrm{~d}, J=8.42 \mathrm{~Hz}, 2 \mathrm{H}), 7.69(\mathrm{~s}, 1 \mathrm{H}), 7.42 \sim 7.39$ (m, 1H), $7.03(\mathrm{~s}, 1 \mathrm{H}), 6.80(\mathrm{~s}, 1 \mathrm{H}), 4.47(\mathrm{~d}, J=5.21 \mathrm{~Hz}$, $2 \mathrm{H}) ;{ }^{13} \mathrm{C}$ NMR $\left(\mathrm{CDCl}_{3}, 101 \mathrm{MHz}\right) \delta: 160.2,157.5,148.9$, $146.8,146.5,139.3,138.9,138.4,136.9,132.8,130.6$, 129.2, 128.0, 126.9, 125.9, 118.2, 114.1, 110.0, 35.3. Anal. calcd for $\mathrm{C}_{20} \mathrm{H}_{12} \mathrm{BrClN}_{6} \mathrm{O}_{2}$ : C 49.66, H 2.50, N 17.37; found $\mathrm{C} 49.50, \mathrm{H} 2.62, \mathrm{~N} 17.23$.

3 -溴-1-(3-氯吡啶-2-基)- $N$ - \{[2-(4-甲基苯基)噁唑-4基]甲基\}- $1 H$-吡唑-5-甲酰胺(11j)：白色固体, 产率 75\%. m.p. $143 \sim 145{ }^{\circ} \mathrm{C} ;{ }^{1} \mathrm{H}$ NMR $\left(\mathrm{CDCl}_{3}, 400 \mathrm{MHz}\right) \delta: 8.46 \sim$ $8.44(\mathrm{~m}, 1 \mathrm{H}), 7.89 \sim 7.86(\mathrm{~m}, 3 \mathrm{H}), 7.56(\mathrm{~s}, 1 \mathrm{H}), 7.39 \sim$ $7.36(\mathrm{~m}, 1 \mathrm{H}), 7.27$ (d, $J=6.02 \mathrm{~Hz}, 2 \mathrm{H}), 6.82(\mathrm{~s}, 1 \mathrm{H}), 4.42$ (d, $J=5.22 \mathrm{~Hz}, 2 \mathrm{H}), 2.41(\mathrm{~s}, 3 \mathrm{H}) ;{ }^{13} \mathrm{C}$ NMR $\left(\mathrm{CDCl}_{3}, 101\right.$ MHz) $\delta: 162.4,157.4,148.9,146.8,141.3,139.2,137.2$, $135.4,129.6,129.6,129.2,127.9,126.4,125.8,124.1$, 110.1, 35.3, 21.6. Anal. calcd for $\mathrm{C}_{20} \mathrm{H}_{15} \mathrm{BrClN}_{5} \mathrm{O}_{2}$ : C 50.82, H 3.20, N 14.82; found C 50.71, H 3.06, N 14.99.

3 -溴-1-(3-氯吡啶-2-基)- $N$-\{ [2-(3-甲基-4-硝基苯基) 噁唑-4-基]甲基 $\}-1 H$-吡唑-5-甲酰胺(11k): 白色固体, 产 率 66\%. m.p. $180 \sim 182{ }^{\circ} \mathrm{C} ;{ }^{1} \mathrm{H} \mathrm{NMR}\left(\mathrm{CDCl}_{3}, 400 \mathrm{MHz}\right)$ $\delta: 8.40 \sim 8.38(\mathrm{~m}, 1 \mathrm{H}), 8.00(\mathrm{~d}, J=8.42 \mathrm{~Hz}, 1 \mathrm{H}), 7.93(\mathrm{~s}$, $1 \mathrm{H}), 7.89 \sim 7.81(\mathrm{~m}, 2 \mathrm{H}), 7.62(\mathrm{~s}, 1 \mathrm{H}), 7.35 \sim 7.32(\mathrm{~m}$, 1H), $7.02(\mathrm{~s}, 1 \mathrm{H}), 6.74(\mathrm{~s}, 1 \mathrm{H}), 4.40(\mathrm{~d}, J=5.61 \mathrm{~Hz}, 2 \mathrm{H})$, 2.61 (s, 3H); ${ }^{13} \mathrm{C}$ NMR $\left(\mathrm{CDCl}_{3}, 101 \mathrm{MHz}\right) \delta: 159.1,156.5$, $149.0,147.9,145.8,138.2,137.4,136.0,133.6,131.1$, $129.5,128.2,127.5,127.0,124.8,124.5,123.7,109.0$, 34.2, 19.5. Anal. calcd for $\mathrm{C}_{20} \mathrm{H}_{14} \mathrm{BrClN}_{6} \mathrm{O}_{4}$ : C 46.40, $\mathrm{H}$ 2.73, N 16.23; found C 46.23, H 2.89, N 16.36.

3 -溴-1-(3-氯吡啶-2-基)- $N$-\{[2-(3-硝基-4-甲基苯基) 噁唑-4-基]甲基\}-1 $H$-吡唑-5-甲酰胺(111)：黄色固体，产 率 68\%. m.p. $197 \sim 199{ }^{\circ} \mathrm{C} ;{ }^{1} \mathrm{H}$ NMR $\left(\mathrm{CDCl}_{3}, 400 \mathrm{MHz}\right)$ $\delta: 8.61(\mathrm{~s}, 1 \mathrm{H}), 8.49 \sim 8.47(\mathrm{~m}, 1 \mathrm{H}), 8.15 \sim 7.89(\mathrm{~m}, 2 \mathrm{H})$, $7.66(\mathrm{~s}, 1 \mathrm{H}), 7.48(\mathrm{~d}, J=8.02 \mathrm{~Hz}, 1 \mathrm{H}), 7.43 \sim 7.40(\mathrm{~m}$, $1 \mathrm{H}), 6.87(\mathrm{~s}, 1 \mathrm{H}), 6.83(\mathrm{~s}, 1 \mathrm{H}), 4.48(\mathrm{~d}, J=5.22 \mathrm{~Hz}, 2 \mathrm{H})$, $2.68(\mathrm{~s}, 3 \mathrm{H}) ;{ }^{13} \mathrm{C} \mathrm{NMR}\left(\mathrm{CDCl}_{3}, 101 \mathrm{MHz}\right) \delta: 160.0,157.5$, $149.6,148.9,146.8,139.3,139.0,137.8,136.3,136.1$, $133.6,130.2,129.2,128.0,126.0,125.9,122.6,110.2$, 35.3, 20.6. Anal. calcd for $\mathrm{C}_{20} \mathrm{H}_{14} \mathrm{BrClN}_{6} \mathrm{O}_{4}$ : C 46.40, H 2.73, N 16.23; found C 46.28, H 2.59, N 16.33.

3 -溴-1-(3-氯吡啶-2-基)- $N$ - \{[2-(2-氟-6-氯苯基)噁唑4-基]甲基 $\}$ - $1 H$-吡唑-5-甲酰胺(11m): 白色固体, 产率 $60 \%$. m.p. $162 \sim 164{ }^{\circ} \mathrm{C} ;{ }^{1} \mathrm{H}$ NMR $\left(\mathrm{CDCl}_{3}, 400 \mathrm{MHz}\right) \delta$ : $8.49 \sim 8.47(\mathrm{~m}, 1 \mathrm{H}), 7.90 \sim 7.87(\mathrm{~m}, 1 \mathrm{H}), 7.76(\mathrm{~s}, 1 \mathrm{H})$, $7.46 \sim 7.38(\mathrm{~m}, 2 \mathrm{H}), 7.34(\mathrm{~d}, J=8.41 \mathrm{~Hz}, 1 \mathrm{H}), 7.20(\mathrm{~s}$, 1H), $7.17 \sim 7.12(\mathrm{~m}, 1 \mathrm{H}), 6.76(\mathrm{~s}, 1 \mathrm{H}), 4.50(\mathrm{~d}, J=5.61$ $\mathrm{Hz}, 2 \mathrm{H}) ;{ }^{13} \mathrm{C} \mathrm{NMR}\left(\mathrm{CDCl}_{3}, 101 \mathrm{MHz}\right) \delta: 162.7,160.2$, $157.5,154.8,148.9,146.8,139.2,137.1,135.3,132.6$, $132.5,129.1,128.0,126.1,125.8,114.9,114.7,110.1$, 35.3. Anal. calcd for $\mathrm{C}_{19} \mathrm{H}_{11} \mathrm{BrCl}_{2} \mathrm{FN}_{5} \mathrm{O}_{2}: \mathrm{C} 44.65, \mathrm{H} 2.17$, $\mathrm{N} 13.70$; found $\mathrm{C} 44.82, \mathrm{H} 2.03, \mathrm{~N} 13.86$.

3 -溴-1-(3-氯吡啶-2-基)- $N$ - $\{[2-(2,4-$ 二氯苯基)噁唑4-基]甲基\}-1 $H$-吡唑-5-甲酰胺(11n): 黄色固体, 产率 $62 \%$. m.p. $167 \sim 169{ }^{\circ} \mathrm{C} ;{ }^{1} \mathrm{H}$ NMR $\left(\mathrm{CDCl}_{3}, 400 \mathrm{MHz}\right) \delta$ : $8.41 \sim 8.39(\mathrm{~m}, 1 \mathrm{H}), 7.81(\mathrm{~d}, J=8.42 \mathrm{~Hz}, 2 \mathrm{H}), 7.62$ (s, 
1H), $7.41(\mathrm{~d}, J=2.02 \mathrm{~Hz}, 1 \mathrm{H}), 7.34 \sim 7.30(\mathrm{~m}, 2 \mathrm{H}), 7.16$ $(\mathrm{s}, 1 \mathrm{H}), 6.74(\mathrm{~s}, 1 \mathrm{H}), 4.41(\mathrm{~d}, J=5.62 \mathrm{~Hz}, 2 \mathrm{H}) ;{ }^{13} \mathrm{C} \mathrm{NMR}$ $\left(\mathrm{CDCl}_{3}, 101 \mathrm{MHz}\right) \delta: 158.2,156.5,147.89,145.8,138.2$, $138.0,136.5,136.2,135.5,132.3,130.7,130.1,128.2$, 127.0, 126.5, 124.8, 123.2, 109.1, 34.1. Anal. calcd for $\mathrm{C}_{19} \mathrm{H}_{11} \mathrm{BrCl}_{3} \mathrm{~N}_{5} \mathrm{O}_{2}$ : C 43.26, $\mathrm{H}$ 2.10, N 13.27; found $\mathrm{C}$ 43.10, H 2.20, N 13.04 .

3 -溴-1-(3-氯吡啶-2-基)- $N$ - $\{[2-(3,5$-二氯苯基)噁唑4-基]甲基 $\}-1 H$-吡唑-5-甲酰胺(110): 黄色固体, 产率 65\%. m.p. $175 \sim 177{ }^{\circ} \mathrm{C}$; ${ }^{1} \mathrm{H}$ NMR $\left(\mathrm{CDCl}_{3}, 400 \mathrm{MHz}\right) \delta$ : $8.48 \sim 8.46(\mathrm{~m}, 1 \mathrm{H}), 7.91 \sim 7.89(\mathrm{~m}, 3 \mathrm{H}), 7.63(\mathrm{~s}, 1 \mathrm{H})$, $7.46 \sim 7.40(\mathrm{~m}, 2 \mathrm{H}), 6.89(\mathrm{~s}, 1 \mathrm{H}), 6.80(\mathrm{~s}, 1 \mathrm{H}), 4.45(\mathrm{~d}, J=$ $5.21 \mathrm{~Hz}, 2 \mathrm{H}) ;{ }^{13} \mathrm{C} \mathrm{NMR}\left(\mathrm{CDCl}_{3}, 101 \mathrm{MHz}\right) \delta$ : 159.6, $157.5,148.9,146.8,139.3,139.0,138.0,136.6,135.8$, $130.6,129.4,129.2$, 128.5, 128.0, 126.0, 125.9, 124.8, 110.2, 35.3. Anal. calcd for $\mathrm{C}_{19} \mathrm{H}_{11} \mathrm{BrCl}_{3} \mathrm{~N}_{5} \mathrm{O}_{2}$ : C 43.26, $\mathrm{H}$ 2.10, N 13.27; found C 43.39, H 2.01, N 13.43.

3 -溴-1-(3-氯吡啶-2-基)- $N$ - $\{[2-(2,6$-二氟苯基)噁唑4-基]甲基 $\}-1 H$-吡唑-5-甲酰胺(11p)：白色固体，产率 $60 \%$. m.p. $153 \sim 155{ }^{\circ} \mathrm{C} ;{ }^{1} \mathrm{H}$ NMR $\left(\mathrm{CDCl}_{3}, 400 \mathrm{MHz}\right) \delta$ : $8.48 \sim 8.46(\mathrm{~m}, 1 \mathrm{H}), 7.90 \sim 7.88(\mathrm{~m}, 1 \mathrm{H}), 7.75(\mathrm{~s}, 1 \mathrm{H})$, $7.51 \sim 7.38(\mathrm{~m}, 2 \mathrm{H}), 7.09 \sim 7.05(\mathrm{~m}, 3 \mathrm{H}), 6.80(\mathrm{~s}, 1 \mathrm{H})$, $4.51(\mathrm{~d}, J=5.61 \mathrm{~Hz}, 2 \mathrm{H}) ;{ }^{13} \mathrm{C} \mathrm{NMR}\left(\mathrm{CDCl}_{3}, 101 \mathrm{MHz}\right) \delta$ : $162.0,159.4,157.5,153.0,148.9,146.8,139.2,139.1$, $137.4,136.8,132.5,132.4,129.1,128.0,125.8,112.5$, 112.2, 110.1, 35.2. Anal. calcd for $\mathrm{C}_{19} \mathrm{H}_{11} \mathrm{BrClF}_{2} \mathrm{~N}_{5} \mathrm{O}_{2}$ : C 46.13, H 2.24, N 14.16; found C 46.30, H 2.38, N 14.03.

辅助材料(Supporting Information) 目标化合物 11a $11 p$ 的核磁共振氢谱和碳谱图谱. 这些材料可以免费从 本刊网站(http://sioc-journal.cn/)上下载.

\section{References}

[1] Guan, A. Y.; Qin, Y. K.; Wang, J. F.; Li, B. J. Fluorine Chem. 2013, $156,120$.

[2] Li, G. Y.; Qian, X. H.; Cui, J. N.; Huang, Q. C.; Zhang, R.; Guan, H. J. Agric. Food Chem. 2006, 54, 125.

[3] Prakash, T. B.; Reddy, G. D.; Padmaja, A.; Padmavathi, V. Eur. J. Med. Chem. 2014, 82, 347.

[4] Ohnmacht, S. A.; Ciancimino, C.; Vignaroli, G.; Gunaratnam, M.; Neidle, S. Bioorg. Med. Chem. Lett. 2013, 23, 5351.

[5] Wang, M. M.; Zhang, Q. Q.; Yue, K.; Li, Q. S.; Xu, F. B. Chin. J.
Org. Chem. 2017, 37, 1774 (in Chinese).

(王梦梦, 张青青, 岳凯, 李庆山, 徐风波, 有机化学, 2017, 37, 1774.)

[6] Zhou, Q.; Zheng, D. D.; Shi, Y. J.; Yao, W.; Qian, H. W.; Ding, Y.; Wei, Z. H.; Shen, A. B.; Feng, X.; Shi, J.; Dai, H. Chin. J. Org. Chem. 2018, 38, 3318 (in Chinese)

(周钱，郑丹丹，石玉军，姚炜，钱宏炜，丁颖，魏中昊，沈爱宝， 冯霞, 石健, 戴红, 有机化学, 2018, 38, 3318.)

[7] Fu, Y.; Zhang, D.; Kang, T.; Guo, Y. Y.; Chen, W. G.; Gao, S.; Ye, F. Bioorg. Med. Chem. Lett. 2019, 29, 570.

[8] Shi, J. J.; Ren, G. H.; Wu, N. J.; Liu, X. H.; Xu. T. M.; Tan, C. X. Chin. J. Org. Chem. 2017, 37, 2131 (in Chinese). (史建俊，任贵华，吴宁捷，刘幸海，许天明，谭成侠，有机化学, 2017, 37, 2131.)

[9] Liu, X. H.; Zhao, W.; Shen, Z. H.; Xing, J. H.; Yuan, J.; Yang, G.; Xu, T. M.; Peng, W. L. Bioorg. Med. Chem. Lett. 2016, 26, 3626.

[10] Liu, X. H.; Zhao, W.; Shen, Z. H.; Xing, J. H.; Xu, T. M.; Peng, W. L. Eur. J. Med. Chem. 2017, 125, 881.

[11] Shi, J. J.; Ren, G. H.; Wu, N. J.; Weng, J. Q.; Xu, T. M.; Liu, X. H.; Tan, C. X. Chin. Chem. Lett. 2017, 28, 1727.

[12] Zhong, L. K.; Jiang, T.; Zhang, F.; Fu, Q.; Liu, X. H.; Xu, T. M.; Ding, C. R.; Chen, J.; Yuan, J.; Tan, C. X. Chin. J. Org. Chem. 2019, 39, 2655 (in Chinese). (钟良坤，江涛，张帆，付庆，刘幸海，许天明，丁成荣，陈杰， 袁静，谭成侠，有机化学, 2019, 39, 2655.

[13] Pirol, S. C.; Caliskan, B.; Durmaz, I.; Atalay, R.; Banoglu, E. Eur. J. Med. Chem. 2014, 87, 140

[14] Song, H. J.; Liu, Y. X.; Xiong, L. X.; Li, Y. Q.; Yang, N.; Wang, Q. M. J. Agric. Food Chem. 2013, 61, 8730.

[15] Gu, B. Q.; Zhu, W. Q.; Fan, W. Z.; Qian, H.; Liu, J. M.; Zhang, A. Q.; Shen, R. X. Modern Agrochem. 2002, 1, 9 (in Chinese). (顾保权，朱伟清，范文政，钱虹，刘建梅，张爱庆，沈荣仙，现 代农药, 2002, 1, 9.)

[16] Fan, W. Z.; Gu, B. Q.; Zhu, W. Q.; Zhang, Y. B. Modern Agrochem. 2005, 4, 9 (in Chinese) (范文政，顾保权，朱伟清，张一宾，现代农药， 2005，4,9.)

[17] Zhou, Y. Y.; Wang, B. L.; Di, F. J.; Xiong, L. X.; Yang, N.; Li, Y. Q.; Li, Y. X.; Li, Z. M. Bioorg. Med. Chem. Lett. 2014, 24, 2295.

[18] Kang, S. H.; Song, B. A.; Wu, J.; He, M.; Hu, D. Y.; Jin, L. H.; Zeng, S.; Xue, W.; Yang, S. Eur. J. Med. Chem. 2013, 67, 14.

[19] Zhu, H. W.; Wang, B. L.; Zhang, X. L.; Xiong, L. X.; Yu, S. J.; Li, Z. M. Chem. Res. Chin. Univ. 2014, 30, 409.

[20] Wang, B. L.; Zhu, H. W.; Li, Z. M.; Wang, L. Z.; Zhang, X.; Xiong, L. X.; Song, H. B. Pest Manage. Sci. 2018, 74, 726.

[21] Ding, C. R.; Pan, Y. Y.; Yin, X.; Tan, C. X.; Zhang, G. F. Chin. J. Org. Chem. 2019, 39, 836 (in Chinese). (丁成荣，潘亚运，殷许，谭成侠，张国富，有机化学，2019，39, 836.)

[22] Dai, H.; Yao, W.; Fang, Y.; Sun, S. Y.; Shi, Y. J.; Chen, J.; Jiang, G Q.; Shi, J. Molecules 2017, 22, 2000.

[23] Huang, P. Q.; Chen, H. Chem. Commun. 2017, 53, 12584.

[24] Zhang, J. F.; Xu, J. Y.; Wang, B. L.; Li, Y. X.; Xiong, L. X.; Li, Y. Q.; Ma, Y.; Li, Z. M. J. Agric. Food Chem. 2012, 60, 7565.

[25] Wang, B. L.; Zhu, H. W.; Ma, Y.; Xiong, L. X.; Li, Y. Q.; Zhao, Y.; Zhang, J. F.; Chen, Y. W.; Zhou, S.; Li, Z. M. J. Agric. Food Chem. 2013, 61, 5483. 